\title{
Abitare un 'immaginario' condiviso. Forme e pratiche collettive di riappropriazione degli spazi urbani
}

\author{
Nicolò Fattori \\ Architetto libero professionista presso lo studio BLUarchitettura, Mestre (VE), Italia \\ nfattori@iuav.it
}

\section{Elena Orsanelli}

Architetto libero professionista presso lo studio Archpiùdue, Vigonza (PD) collaboratrice presso l'Università luav di Venezia, Italia eorsanelli@iuav.it

\section{Sofia Sacchini}

Architetto, collaboratrice presso l'Università luav di Venezia, Italia ssacchini@iuav.it

\begin{abstract}
I sistemi invisibili che governano lo spazio e che trasformano la città suscitano riflessioni e questioni fondative riguardo il progetto, inteso non solo come mera sequenza di azioni, ma come il campo che si crea tra l'agire, gli spazi e le persone. II dispositivo progettuale si colloca all'interno delle maglie del tessuto sociale, nelle pieghe della città, in tempi e spazi che coinvolgono utenti, amministratori, gruppi informali e associazioni. Lo spazio è stato doppiamente indagato: da un punto di vista fisico e da una prospettiva funzionale, ma senza dimenticare l'azione catalizzatrice di attività e pratiche collettive di cui è intrinsecamente portatore. La mappatura degli spazi collettivi e della rete di mobilità attiva esistente è stata associata alla riflessione proposta dal progetto \#tuttamialacittà, ricerche svolte nella regione Veneto ed in particolare nella città metropolitana di Venezia, entrambe interconnesse con l'invisibile, dove è tangibile la necessità di interagire con l'indeterminatezza e l'ambiguità dello spazio, nelle sue molteplici e possibili interpretazioni.
\end{abstract}

\section{Keywords}

Attività sociali, luoghi collettivi, pratiche urbane, rigenerazione, progetto di suolo

\begin{abstract}
The invisible systems that govern the space and transform the city raise reflections and foundational questions about the project, understood not only as a mere sequence of actions, but as the main field that is created between actions, spaces and people. The urban project is located within the social fabric, in the folds of the city, in times and spaces that involve users, administrators, informal groups and associations. The space has been double investigated: from a physical point of view and from a functional perspective, but without forgetting the catalytic action of collective activities and practices of which it is intrinsically bearer. The mapping of the collective spaces and the network of the existing active mobility has been associated with the reflection proposed by the \#tuttamialacittà project. Both researches have been carried out in the Veneto region and in particular in the metropolitan city of Venice, and are interconnected with the invisible, where the need to interact with the indeterminacy and ambiguity of the space is tangible, in its many and possible interpretations.
\end{abstract}

\section{Keywords}

Social activities, collective places, urban practices, regeneration, soil design 
Riflettere sullo spazio pubblico e, più in generale, sulle città contemporanee italiane significa portare alla luce le qualità latenti della vita tra gli edifici. Lo spazio urbano si fa portatore di un'istanza molto pressante negli ultimi decenni: la rivendicazione del diritto allo spazio e alla città, inteso come diritto "alla vita urbana, alla centralità rinnovata, ai luoghi d'incontro e di scambio, ai ritmi di vita e impieghi di tempo che permettano l'uso pieno e intero di questi momenti e luoghi [...]" (Lefebvre, 1968). Urge chiedersi, quindi, quale sia lo spazio in cui si formano i diritti di cittadinanza, quali caratteri spaziali visibili e invisibili che permettano lo sviluppo delle attività sociali, intese come attività che dipendono dalla presenza di altre persone nei medesimi spazi pubblici (Gehl, 2012).

\section{Il territorio degli standard}

I territori del Nord Est, e nello specifico il contesto veneto, si sono dotati nel corso del XX secolo di una considerevole quantità di spazi ed attrezzature del welfare in grado di migliorare il benessere della collettività: scuole, chiese, centri civici e luoghi d'incontro, palestre e spazi per lo sport hanno iniziato a diffondersi nella città contemporanea sviluppando una fitta trama di materiali urbani dotati di peculiari caratteristiche spaziali e tangibili.

La ricerca e le tematiche attorno al concetto di welfare space (Munarin, Tosi, 2014), che si stanno am- pliando ulteriormente durante il nuovo decennio, vedono le attrezzature del welfare come strumenti di coesione sociale, sequenze di spazi che rendono il territorio un luogo confortevole, salubre e sicuro. La costruzione di questi spazi collettivi che, nella maggior parte dei casi, è stata l'esito di disposizioni normative sugli standard urbanistici, ha visto negli ultimi anni una maggior "sensibilizzazione della società rispetto ad istanze ambientali, all'emergere di nuove pratiche sociali" (Tosi, 2014, p.65) e di trasformazione di porzioni di territorio in sistemi capillari di spazi collettivi.

Questo paesaggio, nella sua frammentarietà, è sostenuto da una fitta trama di sistemi e di reti minori, supporto per un "territorio attrezzato e accessibile" (Tosi, 2014, p.64) che fa affidamento su una minuta rete di spazialità attigue, di collegamento, di servizio e di accesso che lo rendono più facilmente fruibile. Le caratteristiche fisiche e spaziali di questi luoghi sono imprescindibili e impongono una serie di limiti e regolamenti indispensabili per il buon funzionamento delle strutture. Al contrario di quello che si è soliti pensare, però, questi luoghi lasciano anche grande spazio all'immaginazione e all'indeterminatezza: è nei pertugi lasciati liberi da imposizioni normative e funzionali che si fa spazio l'idea di una accessibilità per tutti. Spesso accade, infatti, che mentre la fisicità materiale dello spazio rimane 


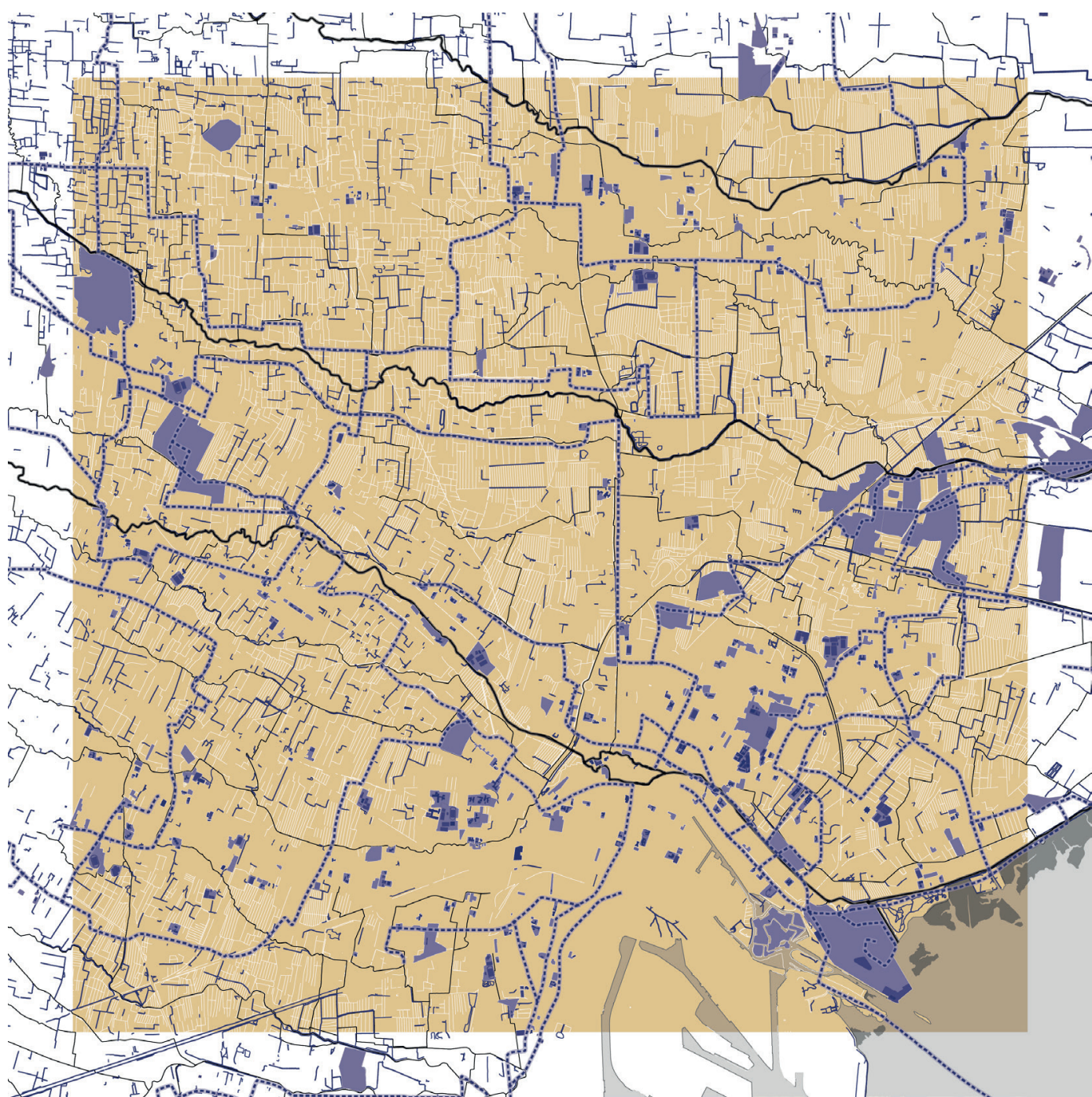

Fig. 1 - Attrezzature del welfare e reticolo minore di strade, sentieri e percorsi legati ad un uso pedonale e ciclabile nell'area metropolitana di Venezia. [Rielaborazione grafica di immagini tratte dal lavoro di ricerca Fare sport fa bene alla città 2: dallo sviluppo e connessione degli spazi sportivo/ricreativi alla definizione di una rete di "mobilità attiva" per la Città Metropolitana di Venezia esito di Protocolli d'intesa tra l'Università luav di Venezia e la Fondazione di Venezia]. (illustrazione: Orsanelli, Fattori, 2021). 
una costante, gli usi e le funzioni mutano con il passare del tempo, con l'evolversi delle necessità o con l'arrivo di nuovi utenti. La realizzazione progettuale di un contesto urbano interviene definendo anche gli usi destinati ad uno spazio, ma quando cambiano le funzioni, quel luogo acquisisce "una nuova tonalità operativa, come una nuova tonalità d'uso" (Von Uexkull, 2013, p.138). Il territorio è, quindi, tanto più accessibile quanto più attrezzato a molteplici tonalità operative, così come vengono descritte le possibilità di utilizzo di uno spazio da Jakob Von Uexkull (Von Uexkull, 2013, p.138): è come se l'utente avesse a disposizione l'elenco delle possibili funzioni progettate per uno spazio, ma fosse lui stesso libero di implementarle nel momento in cui lo utilizza. Lo spazio, nella sua matericità, viene plasmato dai continui cambi d'uso a cui gli utenti lo sottopongono assumendo le sembianze di un luogo inaspettato e includendo l'invisibile tra le sue qualità strutturali: per esempio, ci sono argini progettati per contenere il corso di un fiume che sono diventati lunghi sentieri verdi immersi nella città, ma anche vecchi tracciati ferroviari trasformati in sistemi ciclo-pedonali, parchi pubblici che accolgono funzioni ed attività completamente nuove, come succede per il Parco Albanese di Mestre, interessato saltuariamente da set fotografici per matrimoni e che, in alcuni momenti della giornata, trasforma la sua piazza in un campo da Badminton.

Si può dire, quindi, che il progetto ha molto a che fare con l'invisibile e con l'indeterminato: "progettare per la realtà significa dunque dare spazio a un modo di progettare che fin dall'inizio concepisce l'esistenza di resti, tiene conto del comportamento umano" (Burckhardt, 2019, p.117), della vita quotidiana di coloro che saranno coinvolti nella pianificazione e dal possibile apporto futuro dettato da nuove esigenze ed opportunità. Risulta, quindi, necessario uno sforzo dell'immaginazione che porti all'esplorazione del tempo futuro con l'obiettivo di indicare nuove traiettorie e strategie progettuali di rafforzamento urbano che connettano una visione ampia delle città ad interventi puntuali e mirati distribuiti capillarmente sul territorio.

\section{Leggere il tangibile e l'intangibile dei luoghi}

Nel quadro della riflessione sulla città e sullo spazio pubblico, condotta nell'ambito di un lavoro di ricerca svolto presso l'Università luav di Venezia sul tema Fare sport fa bene alla città 2: dallo sviluppo e connessione degli spazi sportivo/ricreativi alla definizione di una rete di "mobilità attiva" per la Città Metropolitana di Venezia', il contesto metropolitano veneziano rappresenta un terreno d'indagine fertile per l'osservazione di fenomeni e di forme di pratiche collettive. Venezia, sia nella sua accezione di città storica insulare, ma anche di terraferma, infatti, racchiude un potenziale progettuale straordinario per lo sviluppo di spazi, attrezzature urbane e pratiche sociali: un contesto in cui l'azione progettuale dello spazio pubblico assume rilevanza tale da considerarsi vero e proprio dispositivo di conoscenza e rigenerazione urbana per la promozione di scenari futuri.

Attraverso la ricerca, si è cercato di leggere la città e il territorio veneziano come "supporto" per l'innescarsi di azioni di socializzazione, indagando le potenzialità insite con l'obiettivo di perseguire il miglioramento del welfare urbano. II processo di lettura, mappatura e riscrittura del paesaggio, delle attrezzature collettive e dei diversi territori che si susseguono, ha permesso di evidenziare la fisicità e la materialità degli spazi pubblici tipici di questo contesto. È necessario interrogarsi sulle diverse forme della città attraverso un "cambio di prospettiva radicale" (Orsanelli, Sacchini, Fattori, 2021, p.71) per vedere il territorio non solo come stratificazione di materiali urbani, progetti ed elementi tangibili, ma soprattutto come un palinsesto (Corboz, 1998) di azioni e pratiche relazionali. La lettura del territorio veneziano attraverso l'azione del tempo permette di osservare il supporto fisico in chiave di riciclo: il sistema insediativo e i paesaggi della città contempo- 
$\longleftarrow$
$\vdash$
$\sim$
$\mapsto$
$\bullet$
$\bullet$
$\simeq$

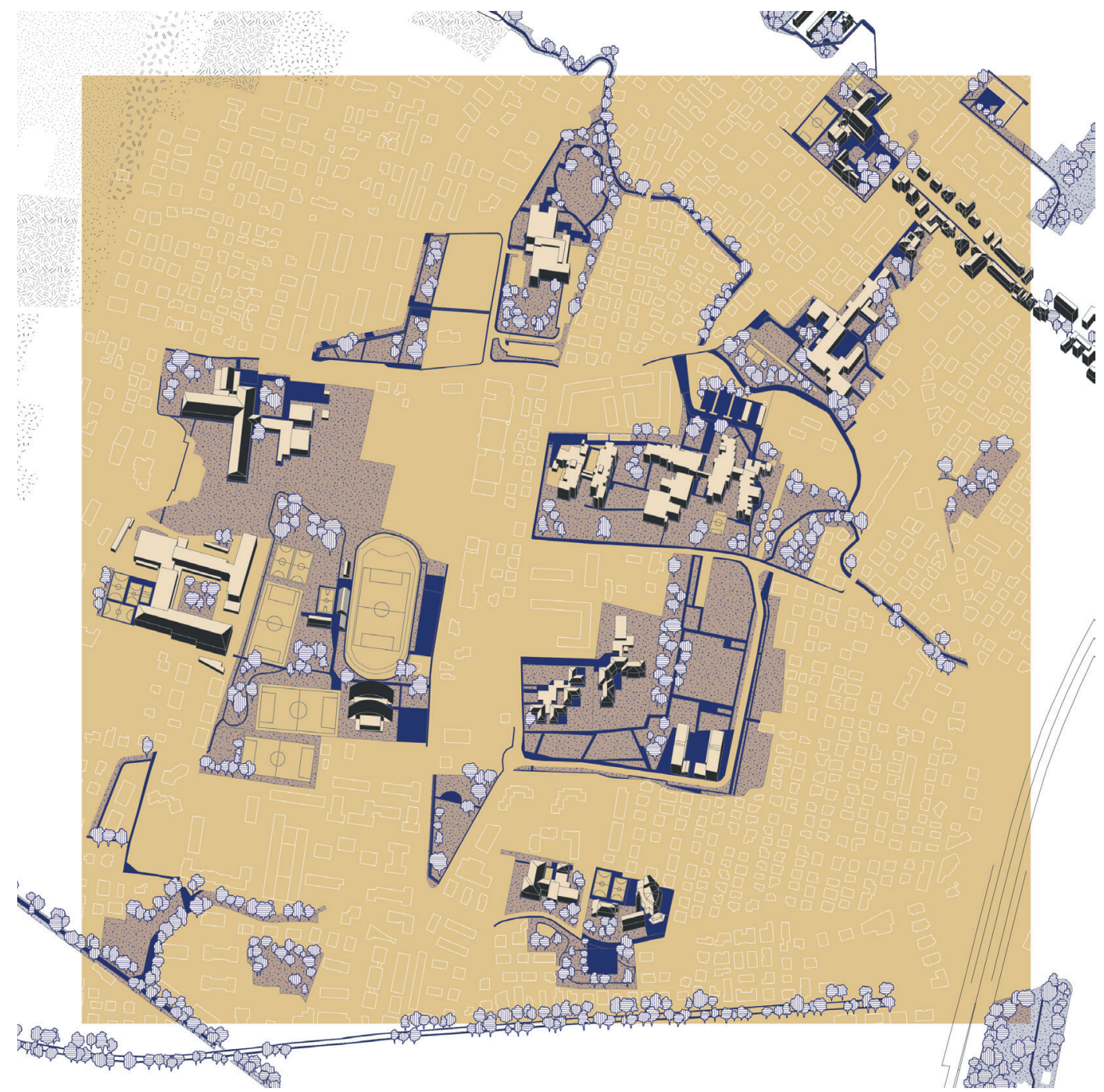

Fig. 2 - Un angolo qualsiasi di mondo: il quartiere Gazzera, Mestre. [Rielaborazione grafica di immagini tratte dal lavoro di ricerca Fare sport fa bene alla città 2: dallo sviluppo e connessione degli spazi sportivo/ricreativi alla definizione di una rete di "mobilità attiva" per la Città Metropolitana di Venezia]. (illustrazione: Orsanelli, Fattori, 2021). 
ranea possono essere visti come materiali capaci di innescare "nuovi cicli di vita" (Fabian, Munarin, 2015, p.16) mediante azioni scandite dal progetto.

La conoscenza di un luogo passa, quindi, attraverso una iniziale ed attenta analisi delle caratteristiche fisico-spaziali esistenti. Tuttavia, rappresentare uno spazio non implica solamente il disegno geometrico dei suoi confini, ma necessita della descrizione degli usi e delle pratiche sociali che vi si svolgono: in quest'ottica, è importante delineare le relazioni umane che si instaurano in uno spazio per riuscire a leggere le interazioni tra processi e progetti in atto. La vita nelle città, le relazioni sociali che si instaurano tra gli edifici, le esperienze tipiche della collettività e le "piccole attività domestiche, come cucire, fare semplici riparazioni, pranzare all'aperto negli spazi pubblici" (Gehl, 2012, p.152) sono azioni "determinate spazialmente e temporalmente, non accadono nel vuoto, ma hanno condizioni [ndr. e conseguenze storiche e fisiche] specifiche" (Inti, 2019, p.33). In questo senso, l'interazione umana porta all'attuazione di un processo di pianificazione territoriale basato sulla condivisione e sulla collaborazione attraverso l'azione congiunta di risorse individuali per la realizzazione di soluzioni a problemi collettivi.

Per superare il rischio di una lettura semplificata, il processo di costruzione di uno scenario progettuale deve passare attraverso l'utilizzo di strumenti di rappresentazione schematici che permettano di delineare i contesti materiali e immateriali dei luoghi. Portare alla luce gli aspetti invisibili del progetto "insegna al visitatore a vedere la sua città, a osservare le sue forme svariate e come esse si ingranano l'una nell'altra" (Lynch, 1964, p.128). Si può dire che l'arte del camminare nel paesaggio (Lanzani, 2011) e la visione della città come luogo d'incontro (Gehl, 2017) offrono una preziosa opportunità per mantenere vivi i contatti tra le persone e il territorio: la gente cammina, si siede e parla, si relaziona con lo spazio aperto attraverso diversi modi tipici dell'abitare insieme.
Pier Luigi Crosta (Tosi, 2006, p. 94), cercando di dare una possibile definizione allo "spazio", nella sua accezione di contesto urbano vissuto, evidenzia come si possa parlare di "posto" in riferimento ad un luogo occupato da qualcuno per una determinata attività, un posto definito come intreccio indissolubile tra spazialità e pratica sociale, un /uogo che viene attraversato da azioni, abitudini quotidiane e pratiche di condivisione.

"Il potere della rappresentazione è quello di far esistere l'espresso, di aprire al pensiero nuove strade, di dischiudere nuove dimensioni, nuovi paesaggi" (Wunenburger, 1999) e andare oltre il mero ridisegno degli oggetti materiali che costruiscono la città, verso la definizione di un immaginario dinamico e vissuto.

\section{Abitare e trasformare uno spazio}

La conoscenza dei luoghi, quindi, passa attraverso l'interazione tra soggetti diversi e i relativi modi di abitare: come afferma Jan Gehl, "una caratteristica comune della vita nello spazio urbano è la versatilità e la complessità delle attività" (Gehl, 2017, p.23) che vi si svolgono, facendo riferimento a tutta quella sfera di attività sociali che definiscono e trasformano nel tempo i caratteri spaziali delle città. Sono molti, per esempio, i capannoni industriali abbandonati riconvertiti in luoghi in cui svolgere attività ludico-ricreative, o le rive dei fiumi concepite come vere e proprie palestre all'aperto che accolgono diversi city users nell'arco della giornata; così come gli ex forti militari diventati, ad oggi, luoghi di riappropriazione comunitaria e collettiva o, ancora, i cosiddetti community hub (Donaggio, 2017), intesi come quei materiali urbani che, sottolineando la relazione persone-comunità, offrono possibili occasioni di manipolazione creativa in chiave di riciclo urbano.

Saper leggere in maniera analitica le diverse attrezzature del welfare presenti nel contesto metropolitano veneziano e, in una visione più ampia, riuscire ad esplorare il supporto urbano e paesaggistico con 
$\varangle$
$\vdash$
$\sim$
$\mapsto$
$\bullet$
$\bullet$
$\curvearrowleft$

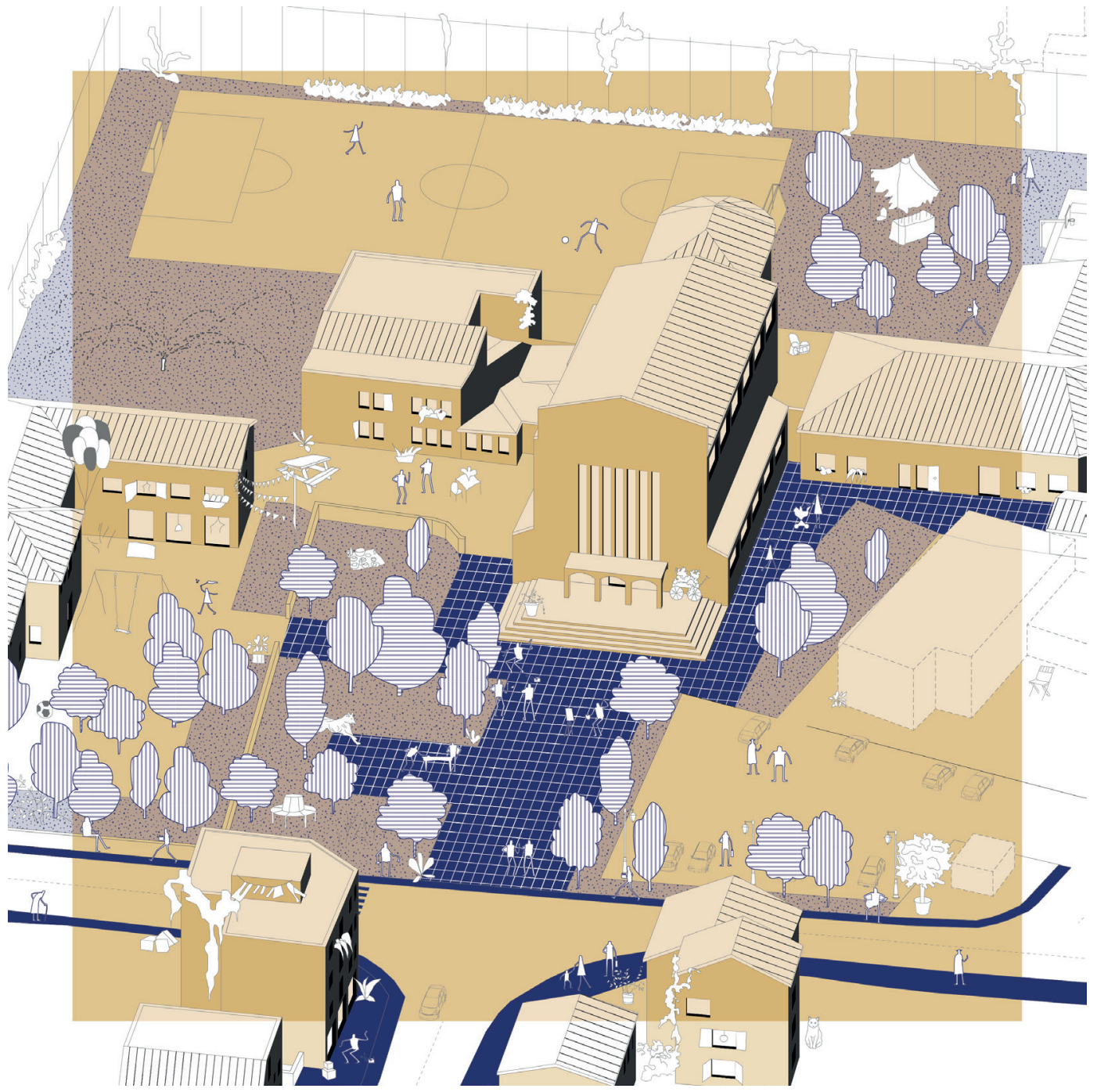

Fig. 3 - La pedonabilità interna: il progetto di suolo come attrattore di azioni e pratiche sociali. [Rielaborazione grafica di immagini tratte dal lavoro di ricerca Fare sport fa bene alla città 2: dallo sviluppo e connessione degli spazi sportivo/ricreativi alla definizione di una rete di "mobilità attiva" per la Città Metropolitana di Venezia]. (illustrazione: Orsanelli, Fattori, 2021). 
uno sguardo attento, porta ad osservare il territorio veneto come un grande laboratorio in cui la trama dei percorsi pubblici e delle economie presenti permette di trasformare le azioni ordinarie, dall'andare a scuola o a lavoro, dal fare sport o svolgere attività tipiche della quotidianità, in concrete esperienze di conoscenza della città. Si finisce per andare a correre su un argine di un fiume invece che sul marciapiede di una strada trafficata, percorrere una carrareccia per arrivare a scuola o fare un pic-nic al parco, evidenziando, attraverso minute azioni abituali, la capillarità degli spazi e delle attrezzature del welfare all'interno della città che ne favoriscono un'abitabilità allargata.

Si può dire, quindi, che l'azione dell'intangibile trasforma lo spazio in un luogo. Luogo inteso non tanto come circoscrizione spaziale con determinati limiti, caratteristiche strutturali ed estetiche, ma come ambito definito tale dal ruolo socio-culturale che le persone gli riconoscono. Con questa accezione, lo spazio assume significato: diventa, quindi, luogo di comunità e incontro, dove eventi e attività si intrecciano, dove "qualcosa succede perché qualcosa succede perché qualcosa succede" (Gehl, 2012, p.99). Lo spazio esiste non solo per i suoi abitanti, ma anche e soprattutto perché i suoi abitanti lo vivono: per questo, il racconto di un luogo comprende sia le caratteristiche in termini dimensionali e materiche dei suoi materiali visibili, sia le "persone che praticano tale spazio e ne fanno esperienza poiché in loro assenza il progetto rimarrebbe invisibile" (Sacchini, 2020, p.43).

Jan Gehl sottolinea come non sia "sufficiente creare spazi dove la gente possa esclusivamente andare e venire: devono esistere condizioni favorevoli per muoversi e soffermarsi in quello spazio" (Gehl, 2012, p.165), devono esistere, quindi, delle ragioni che motivino una sosta, che incentivino le persone a trattenersi in uno spazio e a farne esperienza, deve esistere una meta, una relazione o un utilizzo previsto. Gli elementi invisibili che costituiscono il progetto permettono una lettura del contesto urbano che sotto- linea l'importanza di incentivare nuove forme di ritrovo per l'instaurarsi di una vasta gamma di abitudini collettive, di relazioni sociali, culturali e territoriali durature. Sono gli elementi intangibili e l'aspetto immateriale delle cose che costituiscono la profonda sostanza dei luoghi e dei progetti, sostanza intesa come elemento che sosta, sta, si ferma e agisce.

\section{Un caso studio: Mirano Urla}

A questo proposito, le azioni pilota portate avanti dal Centro Servizi per il Volontariato (CSV) di Venezia in seno al progetto \#tuttamialacittà che ha come obiettivo la promozione del volontariato attivo per la rigenerazione urbana e sociale della città, sono state costitutivamente fondate sul progetto dell'intangibile, inteso come vero e proprio strumento di attivazione urbana, fisica, ma anche e soprattutto sociale; si è passati da "semplici raffigurazioni di un futuro possibile a concreto dispositivo per la gestione del cantiere città" (Secchi, 2000, p.71), del governo dello spazio e della vita che in esso si svolge. II CSV di Venezia in collaborazione con altri centri di servizi per il volontariato della regione Veneto, ha predisposto un percorso formativo-progettuale coinvolgendo le realtà già presenti sul territorio: associazioni culturali e sportive, associazioni di volontariato, enti pubblici, cooperative e gruppi informali. II percorso formativo e poi progettuale realizzato dal CSV di Venezia e dai suoi partner è testimone dell'importanza fondamentale che assume l'idea progettuale ancor prima dell'azione: un pensiero immateriale, indeterminato e ancora acerbo può portare a importanti modifiche del tessuto urbano in chiave fisica e sociale. I protagonisti delle azioni realizzate all'interno del format hanno evidenziato come sia indispensabile sfruttare al meglio le conoscenze e le competenze di tutti i partecipanti e possibili destinatari del progetto, delle associazioni che lavorano sul territorio, degli abitanti, dei professionisti, di giovani e anziani; ognuno di loro ha permesso di "vedere qualcosa di nuovo nei lo- 

ziale, con le contraddizioni, gli elementi peculiari e le stratificazioni degli spazi urbani" (Munarin, 2020, p.14) in cui le azioni hanno preso forma. II fil rouge che definisce il progetto è la volontà di rispondere ad alcuni problemi concreti riscontrati nel paese di Mirano (VE), avendo sempre come fine ultimo la volontà di prendersi cura degli spazi e delle persone che li abitano. Le attività proposte, e poi realizzate grazie ai fondi stanziati dal CSV di Venezia e alla collaborazione tra personale formato e attori sociali, si sono concentrate nell'area del miranese, nell'entroterra orientale di Venezia. II progetto si definisce, quindi, come pensiero-azione "non tanto delle, non solo per, ma con le persone" (Cerrocchi, Dozza, 2018).

\section{Le azioni del progetto}

Le azioni portate avanti dal progetto hanno preso il via con una prima biciclettata esplorativa, che ha permesso ai partecipanti di attraversare il territorio consapevoli dei processi storico-spaziali che ne hanno determinato la conformazione e le funzioni attuali, seguita da un momento di confronto aperto a tutti i cittadini. Per raccogliere le idee e le proposte di tutti è stato organizzato un "open space technology" che si è svolto nel parco Aldo Moro e nei locali del centro

scutere e approfondire le possibili azioni successive e le urgenze particolarmente sentite: hanno partecipato tutti, bambini, anziani, famiglie e giovani, realizzando una sorta di "mappatura collettiva di un futuro possibile" (Sacchini, 2020, p.137). Nei mesi a seguire sono state realizzate le azioni discusse e votate durante il primo incontro tra cui, per esempio, le merende al bosco Parauro per i bambini delle scuole materne che sono state utili da un punto di vista sociale, didattico e formativo in quanto hanno permesso di far conoscere un luogo particolarmente rilevante da un punto di vista ecologico-ambientale e di prendere confidenza con questo spazio, che è uno spazio di tutti. Per coinvolgere i bambini sono stati organizzati dei laboratori di progettazione e realizzazione di giochi che sono stati successivamente collocati all'interno del parco. Per andare incontro all'esigenza più volte espressa di incentivare l'utilizzo di mezzi alternativi alla macchina è stata programmata la "giornata della bicicletta", che ha visto la realizzazione di corsi di autoriparazione del ciclo e lezioni sulla ciclologistica, oltre che la dimostrazione di come alcuni tragitti brevi in città siano facilmente percorribili in bici da chiunque. Da questa necessità è nata anche l'idea di realizzare l'applicazione "Real net Mirano" che evidenzia i percorsi ciclabili consigliati in città e nei territori vicini.

"Le azioni intraprese rispondono ad alcuni problemi concreti quali la cura degli spazi e delle strutture pubbliche spesso in stato di abbandono, la mancanza di luoghi di aggregazione soprattutto per i giovani e mirano a diminuire la solitudine ed altri ostacoli all'inclusione sociale, attraverso l'uso della bicicletta vogliono condividere l'idea di un futuro sostenibile e favorevole all'attività fisica all'aria aperta, anche attraverso la valorizzazione di aree verdi e boschive" (Sacchini, 2020, p.135).

Oltre agli effetti fisici e relazionali riscontrati dai cittadini sulla città, il progetto Mirano Urla ha permesso anche a molte associazioni e realtà presenti sul territorio di creare una rete relazionale densa di significato, aperta e tuttora attiva. 
II progetto, quindi, assume i caratteri di un dispositivo relazionale, riprendendo a questo proposito la definizione di Foucault (Carmagnola, 2015, p.27): un elemento tangibile che induce lo sviluppo di pratiche che hanno peso sia sul piano fisico-spaziale della città, sia sul piano socio-culturale della popolazione. Le attività hanno avuto notevoli implicazioni in termini di dimensioni immateriali e valori simbolici:

"si tratta infatti di esperienze, come si è detto, di ri-
appropriazione della città, che rimettono questi beni
nel ciclo di vita urbana, e innescano processi di inve-
stimento sociale, culturale e personale degli abitanti
in quei beni, mettendo a disposizione energie, tem-
po e competenze molto importanti, ma altresi la ri-
costruzione di un legame, anche affettivo ed empa-
tico, con quei luoghi, e attraverso di essi tra le perso-
ne che vi investono" (Cellamare, 2019, p.142).

Emerge chiaramente, dunque, come l'esplorazione di un territorio vissuto passi inevitabilmente dallo studio di quel substrato intangibile di geografie prossime, vicine ma spesso invisibili proprio perché non riguardano direttamente la matericità degli spazi fisici: conoscere la consistenza spaziale ed immateriale dei luoghi urbani è prerogativa fondamentale per la vita che vi si svolge, per le attività sociali in grado di accogliere ed incentivare processi collettivi. L'azione progettuale, intesa come espressione delle interazioni tra spazi urbani e vita quotidiana, si manifesta tramite l'attivazione di processi ed azioni mirate alla promozione di strategie che incentivano pratiche sociali differenti nei medesimi contesti pubblici. In questa visione, "rendere praticabili gli spazi circostanti una scuola, un parco o un impianto sportivo, in qualche modo fa sì che l'area a contorno dello spazio in cui vi si svolge l'attività principale sia resa disponibile anche a un pubblico più ampio oltre l'utente di quel servizio" (Renzoni, Tosi, 2016, p.35).

È evidente, quindi, che la città fisica, così come il progetto, è costituita anche da elementi intangibili: azioni, abitudini, valori, discorsi, immaginari, rela- zioni, visioni e collaborazioni che non si possono toccare concretamente con mano, contare o catalogare. Tuttavia questi elementi sono fondativi della realtà, sono la sostanza su cui si fonda il progetto e la vita quotidiana. Questi materiali sono sfuggenti e non si possono afferrare concretamente, ma al contempo costituiscono lo scheletro del progetto, il cardine attorno a cui si costruisce il disegno progettuale. Una di queste è certamente la "visione" (Alexander, 1997), che non si basa su calcoli funzionali e categorizzazioni schematiche, ma su un livello di comprensione più profondo. II termine "sostanza" rievoca non solo la matrice costitutiva di questi elementi, ma anche la loro naturale indole nutritiva: avere accesso a un immaginario comune, a una visione futura permette, infatti, di fondare il progetto su un obiettivo finale organico, che non guarda solo alle singole azioni e alla loro applicazione meccanica, ma alla complessità dell'operazione nel suo insieme e alla flessibilità del processo in itinere. Sono tante le questioni che rientrano all'interno della categoria dell'intangibile: il concetto di comunità, la volontà e la ricerca di una soluzione compatibile con il contesto, il desiderio di un miglioramento, le relazioni che si instaurano tra gli abitanti, tra i commercianti e tra gli spazi della collettività. Si costruisce e si conserva un serbatoio invisibile frutto di una conoscenza spaziale e sociale condivisa e di esperienze che hanno lasciato le proprie tracce sui territori coinvolti.

\section{Conclusioni}

È chiaro come il dispositivo progettuale intangibile non risieda tanto nella decisione presa da un singolo soggetto, ma piuttosto nella rete che si intesse tra tutti i soggetti che hanno preso parte all'iniziativa, in qualsiasi ruolo, spazio o tempo, e nella maglia di reIazioni che si sviluppano tra amministrazioni, privati e gruppi con la volontà di orientare il comportamento o il pensiero degli uomini. "Prassi, saperi, misure, istituzioni: siamo chiaramente entro una dimensione esecutiva" (Carmagnola, 2015, p.30) che si col- 
$\varangle$
$\vdash$
$\sim$
$\mapsto$
$\bullet$
$\bullet$
$\simeq$

02

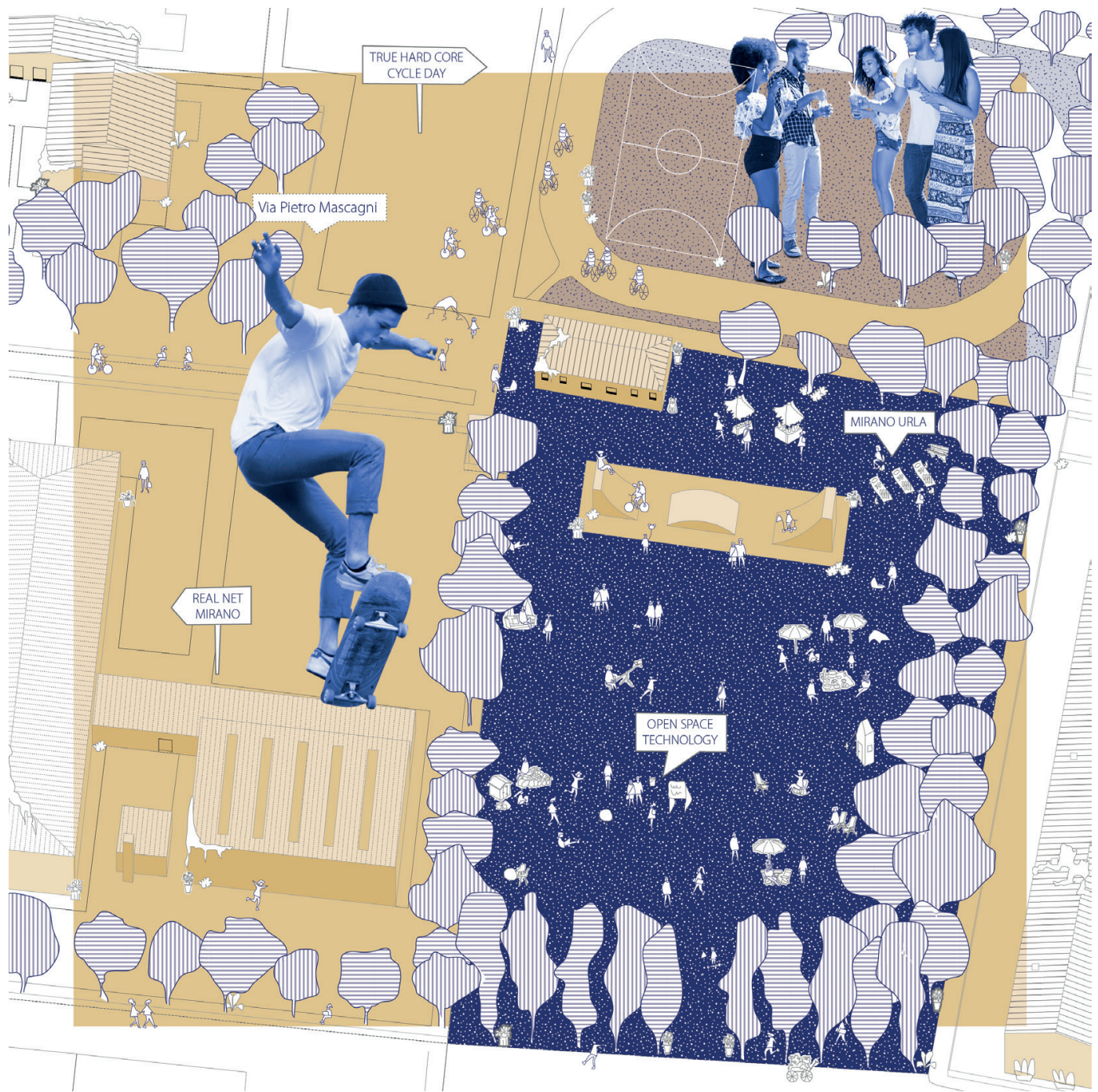

Fig. 4 - II progetto Mirano Urla: espressione della coesione sociale del paese. [Rielaborazione grafica di immagini tratte dal volume: Munarin S., Velo L. (a cura di), Azioni di rigenerazione urbana del volontariato. L'esperienza di \#tuttamialacittà. Progetto di CAVV-CSV di Venezia, Anteferma, Conegliano]. (illustrazione: Sacchini, 2020). 


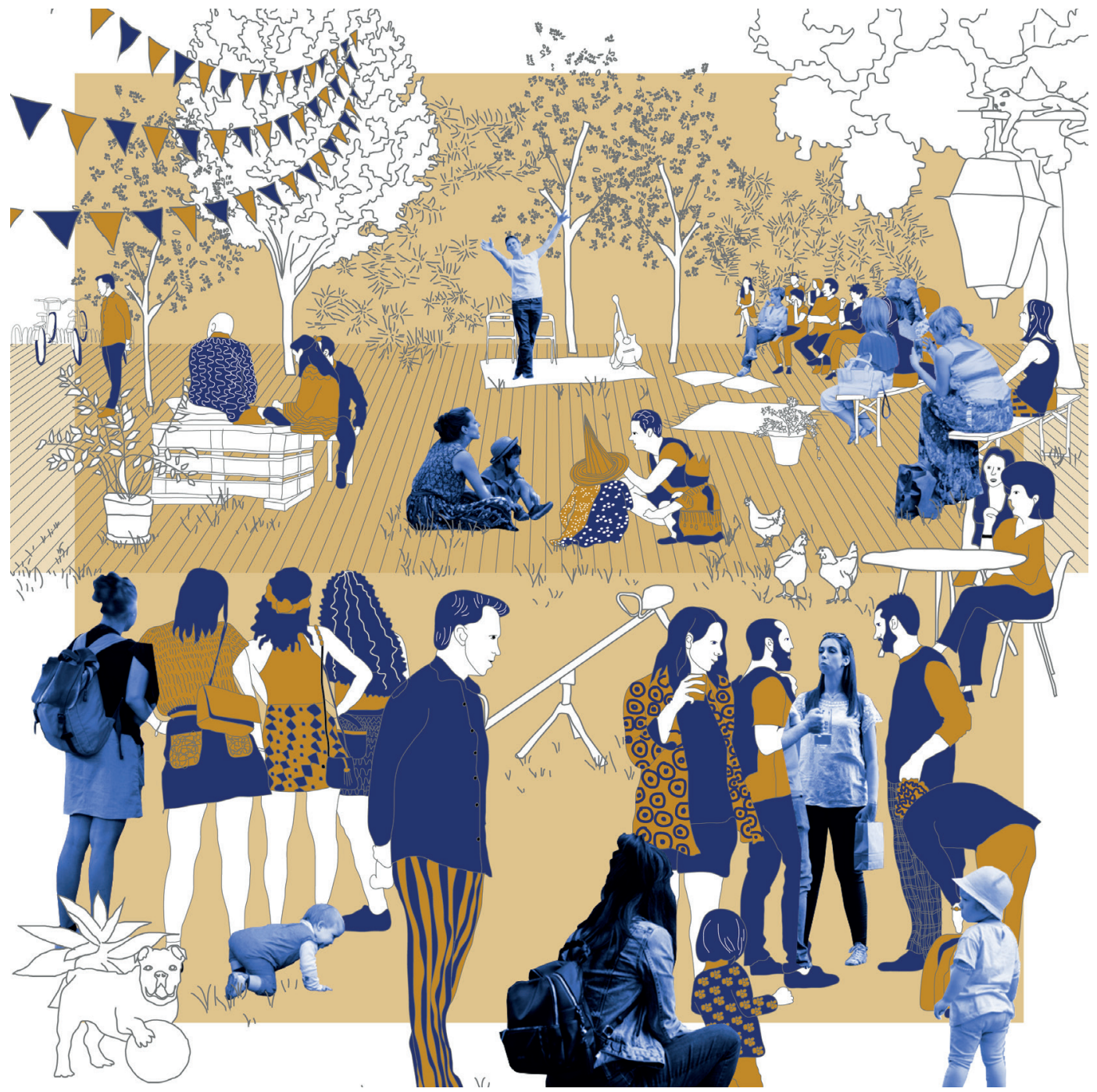

Fig. 5 - Attività di rigenerazione urbana delle associazioni di volontariato in Veneto: la trasformazione della città attraverso il disegno degli spazi e le attività che li animano. [Rielaborazione grafica di immagini tratte dal volume: Munarin S., Velo L. (a cura di), Azioni di rigenerazione urbana del volontariato. L'esperienza di \#tuttamialacittà. Progetto di CAVV-CSV di Venezia, Anteferma, Conegliano]. (illustrazione: Sacchini, 2020). 
loca, quantomeno in parte, all'interno di un contesto prettamente intangibile e talvolta difficilmente descrivibile. Come sostiene Burckhardt, "le cose non sono neutrali, ma esistono" (Burckhardt, 2019, p.111), e di conseguenza condizionano il comportamento, l'atteggiamento, l'agire e, in primis, lo spazio circostante, sia quello fisico che quello mentale. Ne deriva che l'azione di ripensare lo spazio pubblico implica una rilettura in chiave attuale dei contesti urbani già esistenti intesi come luoghi per la collettività e per la condivisione: il processo progettuale di narrazione degli spazi rende i fruitori stessi i veri attori protagonisti che, attraverso la propria esperienza, consentono la vivibilità e la coesione di questi spazi.

Il lavoro di mappatura delle attrezzature collettive e degli spazi del welfare nell'area metropolitana di Venezia, come descritto, ha permesso di portare alla luce il deposito materiale di cui già si dispone, evidenziando le peculiarità e le caratteristiche tipiche di questo territorio. In quest'ottica tenere in considerazione l'orizzonte della visione è fondamentale: "se le persone non riescono a vedere uno spazio, non lo utilizzeranno mai" (Whyte, 1980, p.58). Vedere uno spazio significa riuscire a coglierne i rapporti sociali e a vivere le relazioni interpersonali che si sviluppano intorno e all'interno di quei luoghi. E pro- dimostrato che è possibile progettare tessendo una rete di relazioni di valore che influenza e condiziona la visione che gli abitanti e i partecipanti hanno del paese e di quei luoghi: l'obiettivo perseguito dal progetto, infatti, non è stata la sola e semplice esecuzione di una sequenza di azioni, ma il potenziamento della coesione sociale attraverso la creazione di nuovi luoghi di aggregazione e l'attivazione di processi per un futuro più sostenibile.

Raccontare e progettare per la realtà (Burckhardt, 2019) vuol dire lasciare ampio spazio all'invisibile, ai cittadini, all'indeterminatezza delle funzioni, alla contaminazione, al patrimonio materiale e immateriale (Inti, 2019) della città e della cittadinanza, ai sistemi invisibili che governano il progetto e che trasformano il territorio.

\section{Note}

${ }^{1}$ Ricerca avviata nell'ambito di Protocolli d'intesa tra l'Università luav di Venezia e la Fondazione di Venezia. Gruppo di ricerca: Stefano Munarin (responsabile scientifico), Nicolò Fattori, Elena Orsanelli.

${ }^{2}$ Munarin S., Velo L., (2020), Azioni di rigenerazione urbana del volontariato. L'esperienza di \#tuttamialacittà. Progetto di CAVV-CSV di Venezia, Anteferma, Conegliano. 


\section{Bibliografia}

Alexander C. 1997, Una nuova teoria del disegno urbano, Gangemi Editore, Roma.

Burckhardt L. 2019, II falso è l'autentico. Politica, paesaggio, design, architettura, pianificazione, pedagogia, Quodlibet, Macerata.

Carmagnola F. 2015, Dispositivo. Da Focault al Gadget, Mimesis Edizioni, Milano-Udine.

Cellamare C. 2019, Città fai-da-te. Tra antagonismo e cittadinanza. Storie di autorganizzazione, Donzelli Editore, Roma-Bari.

Cerrocchi L., Dozza L. 2018, Contesti educativi per il sociale. Progettualità, professioni e setting per il benessere individuale e di comunità, Franco Angeli Edizioni, Milano.

Corboz A. 1998, II territorio come palinsesto, in Viganò P. (a cura di), Ordine sparso. Saggi sull'arte, il metodo, la città e il territorio, Franco Angeli Edizioni, Milano, pp. 22-27.

Donaggio E. 2017, Sport e rigenerazione urbana: ripensare gli impianti sportivi, Arcipelago, Milano, <https://www. arcipelagomilano.org/archives/45609> (10/21).

Fabian L., Munarin S. 2015, Re-cycle Veneto, in L. Fabian, S. Munarin, E. Donadoni (a cura di), Re-cycle Veneto, Aracne Editrice, Ariccia, pp. 11-23.

Gehl J. 2012, Vita in città. Spazio urbano e relazioni sociali, Maggioli Editore, Sant'Arcangelo di Romagna.

Gehl J. 2017, Città per le persone, Maggioli Editore, Sant'Arcangelo di Romagna.

Inti I. 2019, Pianificazione aperta. Disegnare e attivare processi di rigenerazione territoriale, in Italia, LetteraVentidue Edizioni, Siracusa.

Lanzani A. 2011, In cammino nel paesaggio, Carocci Editore, Roma.
Lefebvre H. 1968, Le droit à la ville, Editions Anthropos, Parigi.

Lynch K. 1964, L'immagine della città, Marsilio Editori, Venezia.

Munarin S., Tosi M.C. 2014, Welfare space. On the role of welfare state policies in the construction of the contemporary city, LISt Lab, Italia.

Orsanelli E., Sacchini S., Fattori N. 2021, Vuoti apparentie vuoti reali: abitare spazi dimenticati. Riflessioni e sguardi sul territorio contemporaneo, «Officina», n. 32, pp. 68-71.

Renzoni C., Tosi M.C. 2016, Oltre gli standard. Per un territorio attrezzato e accessibile, in Raggruppamento di ricerca "NUQ - New Urban Question" (a cura di), Un manifesto per il Veneto. Scenari, obiettivi, azioni, Mimesis Edizioni, Sesto San Giovanni, pp. 31-35.

Sacchini S. 2020, Rappresentare la rigenerazione urbana e l'innovazione sociale, in Munarin S., Velo L. (a cura di), Azioni di rigenerazione urbana del volontariato. L'esperienza di \#tuttamialacittà. Progetto di CAVV-CSV di Venezia, Anteferma, Conegliano, pp. 43-47.

Secchi B. 2000, Prima lezione di urbanistica, Laterza Editori, Roma.

Tosi M.C. 2014, Veneto attrezzato e accessibile, in Fabian L. (a cura di), New Urban Question. Ricerche sulla città contemporanea 2009-2014, Aracne Editrice, Roma, pp. 66-75. Whyte W. H. 1980, The Social Life of Small Urban Spaces, Conservation Foundation, Washington D.C.

Wunenburger, J.-J. 1999, Filosofia delle immagini, Piccola biblioteca Einaudi, Torino. 